\title{
The COVID-19 Pandemic and Cancer Surgery
}

\author{
C. S. Pramesh ${ }^{1} \cdot$ Rajendra Achyut Badwe ${ }^{1}$
}

Published online: 4 November 2021

(C) Indian Association of Surgical Oncology 2021

The past year and a half has posed serious challenges to healthcare systems in all parts of the world. The COVID-19 pandemic has spread globally, with 221 countries having been affected, 224 million people infected, and 4.6 million deaths as on 14th September 2021 [1]. Different countries responded in different ways, with varying degrees of restrictions and lockdowns. While the pandemic is certainly unprecedented by the extent of spread, the duration that it has lasted with multiple waves, the impact that it has had on other areas of healthcare are less well recognized. Maternal health and childbirth, immunization programs, and care of infectious diseases like HIV and tuberculosis as well as noncommunicable diseases like diabetes, pulmonary disease, and cancer have all been impacted to varying degrees. During the initial phase of the pandemic, this was predominantly due to widespread fear amongst healthcare providers and the public, combined with stringent travel restrictions. It is debatable whether many of these responses were commensurate with the stage of the pandemic. For example, in India, the fear and the stringency of restrictions were maximum at the beginning, when the number of patients infected with COVID-19 was not very high. Nevertheless, many healthcare providers had either shut down their services or scaled them down considerably; many more were converted to COVID-19 treatment facilities to cope with the increasing number of patients who tested positive. Overall, there were considerable reductions in the number of non-COVID-19 patients treated at medical centers. The long-term impact of these reductions is not yet known.

Several studies have documented the fact that cancer care was impacted considerably during the pandemic [2-4]. A representative study [2] conducted in 41 centers of the National Cancer Grid in India found that there were substantial reductions in the number of patients treated at

Rajendra Achyut Badwe

badwera@tmc.gov.in

1 Tata Memorial Centre, Homi Bhabha National Institute, Dr Ernest Borges Marg, Parel, Mumbai 400012, India these centers. New patient registrations dropped by $54 \%$, follow-up visits by $46 \%$, hospital admissions by $36 \%$, daycare chemotherapy by $37 \%$, major surgeries by $49 \%$, minor surgeries by $52 \%$, radiation treatment by $23 \%$, diagnostic pathology tests by $38 \%$, radiological tests by $43 \%$, and palliative care referrals by $29 \%$. These reductions happened during the peak of the national lockdown (March to May 2020) and have, to a large extent, returned to normal. However, the impact of lack of access to cancer care during these months could potentially result in diagnoses at more advanced stages and poorer long-term cancer outcomes. The reductions in surgeries in the NCG study is not restricted to India alone-between March and August 2020, there was a mean $63.6 \%$ decrease in operating theatre capacity and $27.3 \%$ reduction in surgical staffing in the UK [5]; similar reductions were seen in other countries like Japan and Spain $[4,6]$. A global modeling study estimated that over 28 million surgeries were postponed or cancelled during the peak 12 weeks of the pandemic, of which there were likely to be over 2.3 million cancer surgeries [7]. Surgery plays a critical role in cancer management, and reductions in surgical volumes, or prolonged delays in cancer surgery for any reason are likely to have a detrimental effect on cure rates [8].

Early reports from China and Italy fuelled fear of poor outcomes with cancer management during the COVID-19 pandemic [9]. This led to many organizations and professional societies modifying their management protocols $[10$, 11] to adopt a more conservative approach with cancer treatment. However, as the pandemic progressed, increasing data from several groups suggested that with standard infection prevention and control practices, perioperative outcomes were not worse than in the pre-pandemic era $[12,13]$. In approximately 500 major cancer surgeries performed during the first wave, the overall postoperative morbidity and mortality was similar to pre-COVID periods [12]. This was achieved by a combination of administrative and medical measures including screening patients and relatives outside the hospital for symptoms and signs of COVID-19, segregating those with symptoms for testing, minimizing crowds within the hospital, routine preoperative testing 
for SARS-CoV-2, and the use of universal precautions for staff $[14,15]$. These results are likely to be even better with COVID-19 vaccination being increasingly available in many countries. In fact, there is strong data to support that elderly patients planned for cancer surgery were the subgroup most likely to benefit with a number needed to vaccinate (NNV) being 351 (best case, 196, worst case 816) compared to the general population, where the NNV was 1840 (best case 1196, worst case 3066) [16].

We now have reasonable data on patients operated after testing positive for SARS-CoV-2 based on which some recommendations can be made regarding routine preoperative testing and the timing of surgery after infection with SARS$\mathrm{CoV}-2$. An international cohort study demonstrated that routine preoperative testing was beneficial in major surgeries areas of high SARSCoV-2 prevalence, while it was not useful for minor surgeries in low SARS-CoV-2 prevalence areas. The largest data available on the optimum timing of surgery after prior COVID infection is from the COVIDSurg collaborative which evaluated over 140,000 patients from 116 countries, of which 3127 were operated after testing positive for SARS-CoV-2. While the adjusted postoperative mortality in those with prior COVID-19 operated $>7$ weeks after their COVID diagnosis was similar to those without SARS-CoV-2 infection (adjusted odds ratio [OR], 1.02), it was significantly higher when patients were operated 0-2 weeks (adjusted OR, 3.22), 3-4 weeks (adjusted OR, 3.03), and after infection with SARS-CoV-2. Based on this data, there seems to be a case for delaying surgery by 6 weeks after first diagnosis of COVID-19; however, this needs to be balanced against the risks of delaying cancer surgery and disease progression. The postoperative mortality in patients who develop postoperative SARS-CoV-2 infection is much higher than those without (head and neck cancer surgery, $10.3 \%$ vs $0.3 \%$ [17]; colorectal cancer surgery, adjusted OR, 16.9 [18]), clearly emphasizing the need for meticulous attention to infection prevention and control practices.

We now know much more about COVID-19 than we did in 2020, and with adequate precautions, risk to both hospital staff and patients undergoing cancer surgery can be minimized to a large extent. Symptomatic screening for symptoms of COVID-19 in all patients, strict segregation of patients with suspected symptoms, low threshold for testing, routine preoperative testing for SARS-CoV-2, and standard precautions for operating room staff are all measures that can be easily implemented. Additionally, with vaccination being available to the vast majority of the population, it is expected that even if patients and staff are infected, it is likely to result in mild disease without serious consequences. Healthcare workers, patients, and their relatives should be actively encouraged to get vaccinated, thereby reducing overall risks in the population and in hospital. Routine preoperative testing for SARS-CoV-2 in high-incidence areas, combined with meticulous infection prevention and control practices perioperatively, will minimize risks to both patients and hospital staff. There is now increasing data that failure to prioritize cancer care will lead to delayed diagnoses, more advanced cancers, and deferred treatment, all of which have a direct adverse impact on long-term cancer outcomes. We therefore strongly advocate the continuation of cancer care even during pandemics, unless the healthcare system is completely overwhelmed.

\section{Declarations}

Conflict of Interest The authors declare that they have no conflict of interest.

\section{References}

1. World Health Organization. WHO Coronavirus Disease (COVID19) Dashboard http://covid19.who.int (Accessed on 15 Sept 2021)

2. Ranganathan P, Sengar M, Chinnaswamy G, Agrawal G, Arumugham R, Bhatt R, et al (2021) National Cancer Grid of India. Impact of COVID-19 on cancer care in India: a cohort study. Lancet Oncol 22: 970-976

3. John N, Wang GM, Cioffi G, Waite K, Patil N, Kyasaram R et al (2021) The negative impact of the COVID-19 pandemic on oncology care at an academic cancer referral center. Oncology (Williston Park) 35:462-470

4. Tokunaga M, Yoshikawa T, Boku N, Nishida Y, Tanahashi T, Yamada T, et al (2021) Impact of COVID-19 on gastric cancer treatment in Japanese high-volume centers: a JCOG stomach cancer study group survey. Surg Today 1-8

5. Beatty JW, Clarke JM, Sounderajah V, Acharya A, Rabinowicz S, Martin G, et al (2021) PANSURG-PREDICT Collaborative. Impact of the COVID-19 pandemic On Emergency Adult Surgical Patients and Surgical Services: An International Multi-Center Cohort Study and Department Survey. Ann Surg. https://doi.org/ 10.1097/SLA.0000000000005152.

6. Martínez-Hernández NJ, Caballero Silva U, Cabañero Sánchez A, Campo-Cañaveral de la Cruz JL, Obeso Carillo A, Jarabo Sarceda JR, On Behalf Of The Scientific Committee Of The Spanish Thoracic Surgery Society (2021) Effect of COVID-19 on Thoracic Oncology Surgery in Spain: A Spanish Thoracic Surgery Society (SECT) Survey. Cancers (Basel) 13: 2897

7. COVIDSurg Collaborative (2020) Elective surgery cancellations due to the COVID-19 pandemic: global predictive modelling to inform surgical recovery plans. Br J Surg 107: 1440-1449

8. Garcia D, Siegel JB, Mahvi DA, Zhang B, Mahvi DM, Camp ER et al (2020) What is Elective Oncologic Surgery in the Time of COVID-19? A Literature Review of the Impact of Surgical Delays on Outcomes in Patients with Cancer. Clin Oncol Res 3:1-11

9. Zhang L, Zhu F, Xie L, Wang C, Wang J, Chen R et al (2020) Clinical characteristics of COVID-19-infected cancer patients: a retrospective case study in three hospitals within Wuhan. China Ann Oncol 31:894-901

10. ASCO. ASCO coronavirus resources. Available from: https:// www.asco.org/sites/new-www.asco.org/files/content-files/2020ASCO-Guide-Cancer-COVID19.pdf. Accessed 13 Sept 2021 
11. ESMO. ESMO COVID-19 and cancer. Available from: https:// www.esmo.org/guidelines/cancer-patient-management-duringthe-covid-19-pandemic. Accessed 13 Sept 2021

12. Shrikhande SV, Pai PS, Bhandare MS, Bakshi G, Chaukar DA, Chaturvedi P et al (2020) Outcomes of Elective Major Cancer Surgery During COVID 19 at Tata Memorial Centre: Implications for Cancer Care Policy. Ann Surg 272:e249-e252

13. Glasbey JC, Nepogodiev D, Simoes JFF, Omar O, Li E, Venn ML (2021) COVIDSurg Collaborative. Elective Cancer Surgery in COVID-19-Free Surgical Pathways During the SARS-CoV-2 Pandemic: An International, Multicenter, Comparative Cohort Study. J Clin Oncol 39: 66-78

14. The Tata Memorial Centre COVID-19 Working Group (2020) The COVID-19 pandemic and the Tata Memorial Centre response. Ind J Cancer 57: 123-8

15. Pramesh CS, Badwe RA (2020) Cancer management in India during COVID-19. N Engl J Med 382: e61
16. COVIDSurg Collaborative, GlobalSurg Collaborative (2021) SARS-CoV-2 vaccination modelling for safe surgery to save lives: data from an international prospective cohort study. Br J Surg 2021:znab101. https://doi.org/10.1093/bjs/znab101

17. COVIDSurg Collaborative (2021) Head and neck cancer surgery during the COVID-19 pandemic: An international, multicenter, observational cohort study. Cancer. 127:2476-2488

18. COVIDSurg Collaborative (2020) Outcomes from elective colorectal cancer surgery during the SARS-CoV-2 pandemic. Colorectal Dis. https://doi.org/10.1111/codi.15431

Publisher's Note Springer Nature remains neutral with regard to jurisdictional claims in published maps and institutional affiliations. 